\title{
Clinical predictors of long-term survival in newly diagnosed transplant eligible multiple myeloma - an IMWG Research Project
}

Saad Z. Usmani ${ }^{1}$, Antje Hoering ${ }^{2}$, Michele Cavo ${ }^{3}$, Jesus San Miguel ${ }^{4}$, Hartmut Goldschimdt ${ }^{5}$, Roman Hajek ${ }^{6}$, Ingemar Turesson ${ }^{7}$, Juan Jose Lahuerta ${ }^{8}$, Michel Attal ${ }^{9}$, Bart Barlogie $^{10}$, Jae Hoon Lee ${ }^{11}$, Shaji Kumar $\mathbb{D}^{12}$, Stig Lenhoff ${ }^{13}$, Gareth Morgan ${ }^{14}$, S. Vincent Rajkumar $\mathbb{B}^{15}$, Brian G. M. Durie ${ }^{16}$ and Philippe Moreau ${ }^{17}$

\begin{abstract}
Purpose: multiple myeloma is considered an incurable hematologic cancer but a subset of patients can achieve longterm remissions and survival. The present study examines the clinical features of long-term survival as it correlates to depth of disease response. Patients \& Methods: this was a multi-institutional, international, retrospective analysis of high-dose melphalan-autologous stem cell transplant (HDM-ASCT) eligible MM patients included in clinical trials. Clinical variable and survival data were collected from 7291 MM patients from Czech Republic, France, Germany, Italy, Korea, Spain, the Nordic Myeloma Study Group and the United States. Kaplan-Meier curves were used to assess progression-free survival (PFS) and overall survival (OS). Relative survival (RS) and statistical cure fractions (CF) were computed for all patients with available data. Results: achieving CR at 1 year was associated with superior PFS (median PFS 3.3 years vs. 2.6 years, $p<0.0001$ ) as well as OS (median OS 8.5 years vs. 6.3 years, $p<0.0001$ ). Clinical variables at diagnosis associated with 5-year survival and 10-year survival were compared with those associated with 2-year death. In multivariate analysis, age over 65 years (OR 1.87, $p=0.002$ ), IgA Isotype (OR 1.53, $p=0.004$ ), low albumin $<3.5 \mathrm{~g} / \mathrm{dL}$ $(\mathrm{OR}=1.36, p=0.023)$, elevated beta 2 microglobulin $\geq 3.5 \mathrm{mg} / \mathrm{dL}$ (OR 1.86, $p<0.001)$, serum creatinine levels $\geq 2 \mathrm{mg} /$ $\mathrm{dL}(\mathrm{OR} 1.77, p=0.005)$, hemoglobin levels $<10 \mathrm{~g} / \mathrm{dL}$ (OR 1.55, $p=0.003)$, and platelet count $<150 \mathrm{k} / \mu \mathrm{L}(\mathrm{OR} 2.26, p<$ 0.001 ) appeared to be negatively associated with 10-year survival. The relative survival for the cohort was $\sim 0.9$, and the statistical cure fraction was $14.3 \%$. Conclusions: these data identify CR as an important predictor of long-term survival for HDM-ASCT eligible MM patients. They also identify clinical variables reflective of higher disease burden as poor prognostic markers for long-term survival.
\end{abstract}

\section{Introduction}

The last decade has witnessed major progress in clinical outcomes in multiple myeloma (MM), attributable to the introduction of several novel agents which, when

\footnotetext{
Correspondence: Saad Z. Usmani (saad.usmani@atriumhealth.org) ${ }^{1}$ Hematogic Oncology and Blood Disorders, Levine Cancer Institute/Atrium Health, Charlotte, NC, United States

${ }^{2}$ Cancer Research and Biostatistics, Seattle, Washington, United States

Full list of author information is available at the end of the article.
}

combined with either each other or conventional cytotoxic drugs, have imparted a high frequency of complete responses $(\mathrm{CR})^{1-4}$. This is especially true for younger and high-dose melphalan-autologous stem cell transplant (HDM-ASCT) eligible patients where pre-transplant induction therapy is able to provide $>60 \%$ rates of very good partial response or better. HDM-ASCT further deepens the response and results in $\sim$ CR rates. Several prospective studies have reported strong correlation 
between depth of response (achieving CR) and progression-free survival (PFS) and overall survival (OS) in the era of novel agents. There is also a clear recognition that patients achieving CR may have variable $\mathrm{PFS} / \mathrm{OS}^{5}$, perhaps dictated by differences in disease biology and shortcomings of the current response criteria. Minimal residual disease (MRD) testing, by either flow or DNA sequencing, has demonstrated that $\mathrm{CR}$ patients may have residual disease (MRD positive) putting them at risk for early relapse ${ }^{6}$.

These advances have re-ignited the debate on possible functional curability of a subset MM patients ${ }^{5}$. The Spanish Myeloma group recently reported the strong correlation of depth of response to higher likelihood of long-term PFS/OS (12-year PFS was 28\% in CR, 19\% in nCR, $10 \%$ in VGPR and 11\% in PR groups; 12 -year OS was $35 \%$ in CR, $22 \%$ in nCR, $16 \%$ in VGPR and $16 \%$ in PR groups) among 344 post HDM-ASCT patients followed for a median of nearly 13 years ${ }^{7}$. The current study is a collaborative effort by the Internal Myeloma Working Group to closely examine the clinical predictors of longterm survival in $\mathrm{MM}(>10 \mathrm{yr})$ in terms of their presenting features, quality of response and management in order to guide therapeutic investigations aimed at long-term disease control.

\section{Patients and methods Patient population}

A total of 7291 patients with survival data were considered for the analysis, age limit up to 75 years. These patients come from the following nations: Czech Republic, France, Germany, Italy, South Korea, Spain, the Nordic Myeloma Study Group (Sweden, Denmark, Norway), and the United States. Tables comparing patient characteristics for several key variables for patients meeting specified characteristics are included, as are tables showing a summary of available data by country (Supplemental Table 3). It is important to note that over $90 \%$ of the patients in the dataset were from the pre-novel therapy induction era and $\sim 10 \%$ did received thalidomide as part of their upfront therapy (Total Therapy 2 thalidomide arm, GMMG-HD3 thalidomide arm and BO2002).

\section{Statistical analysis}

Kaplan-Meier curves $^{8}$ were used to assess OS, PFS, and CR duration (where applicable). Relative survival (RS) ${ }^{9}$ was computed for all patients with available data. The goal of RS analysis is to compare the survival of a cohort with a certain disease to the survival of the general population with similar characteristics to that cohort. We estimate the ratio of observed survival amongst patients with a certain condition to expected survival in a comparable population with comparable characteristics (such as nationality, age, and sex) to those patients. In this analysis, we consider a patient's nationality, age at enrollment, and sex (where available), and obtained expected survival estimates from life tables for a person with those characteristics starting in the year of the patient's enrollment (note: where sex data not available, we use data from life tables estimated for nationality and age which did not adjust for sex). Here, we provide estimates for RS for the IMWG data overall and by individual country, with point estimates at each 1-year interval from start of therapy, and a 95\% confidence band for the RS.

Berkson-Gage cure modeling ${ }^{10}$ was performed for a proportion of patients who represent a "cure proportion;" in other words, these patients are said to have survival according to the normal survival rates for a person of that age/sex/nationality. The figures in the manuscript represent survival curves, where survival is the survival for the "cure proportion" plus the survival for the remainder of patients.

\section{Results}

The OS, PFS, and CR duration separated by each country is presented in the supplemental files (Supplemental Figure 1a-d). For the combined dataset analyses, the OS and PFS were compared between patients who achieved CR landmarked at 1 year after diagnosis (Fig. 1) to examine the effects of depth of response on outcome. Patients who achieved CR had a superior PFS (median PFS 3.3 years vs. 2.6 years, $p<0.0001$ ); this effect was most pronounced for the patients who received thalidomide (the only novel agent used in dataset) with higher CR rates observed and median PFS of 6 years for those who achieved CR (Supplemental Figure 1e/f). The benefit was also seen in terms of OS for the whole group (median OS 8.5 years vs. 6.3 years, $p<0.0001$ ). Patients who had albumin $<3.5 \mathrm{~g} / \mathrm{dL}(p=0.002)$ or an IgG isotype $(p<$ $0.001)$ were less likely to achieve a $C R$ at the 1-year landmark (Table 1). Age over 65 years was not associated with inability to achieve $\mathrm{CR}$ in this dataset. We further examined clinical variables associated with sustained $C R$ for 3 years and 5 years in subset of patients where these data were available (Supplemental Tables 6, 7) using logistic regression. These analyses were not very informative, as only IgA isotype, light chain only disease and BMPC $>30 \%$ featured with statistical significance in multivariate analyses for both time points.

We also examined the clinical variables associated with 10-year survival compared with 2-year death (Table 2). In a univariate model, age $\leq 65$ years (OR 2.24, $p<0.001$ ), normal albumin (OR 2.01, $p<0.001$ ), low beta 2 microglobulin $\leq 3.5 \mathrm{mg} / \mathrm{dl}$ (OR 3.51, $p<0.001$ ), serum creatinine levels $\leq 2 \mathrm{mg} / \mathrm{dL}$ (OR 3.73, $p<0.001$ ), hemoglobin levels $\geq 10 \mathrm{~g} / \mathrm{dL} \quad(\mathrm{OR} 2.98, p<0.001)$, platelet count $\geq$ $150 \mathrm{k} / \mu \mathrm{L}$ (OR 3.94, $p<0.001$ ), normal serum LDH levels (OR $2.78, p<0.001$ ), no cytogenetic abnormality (OR 3.21, 

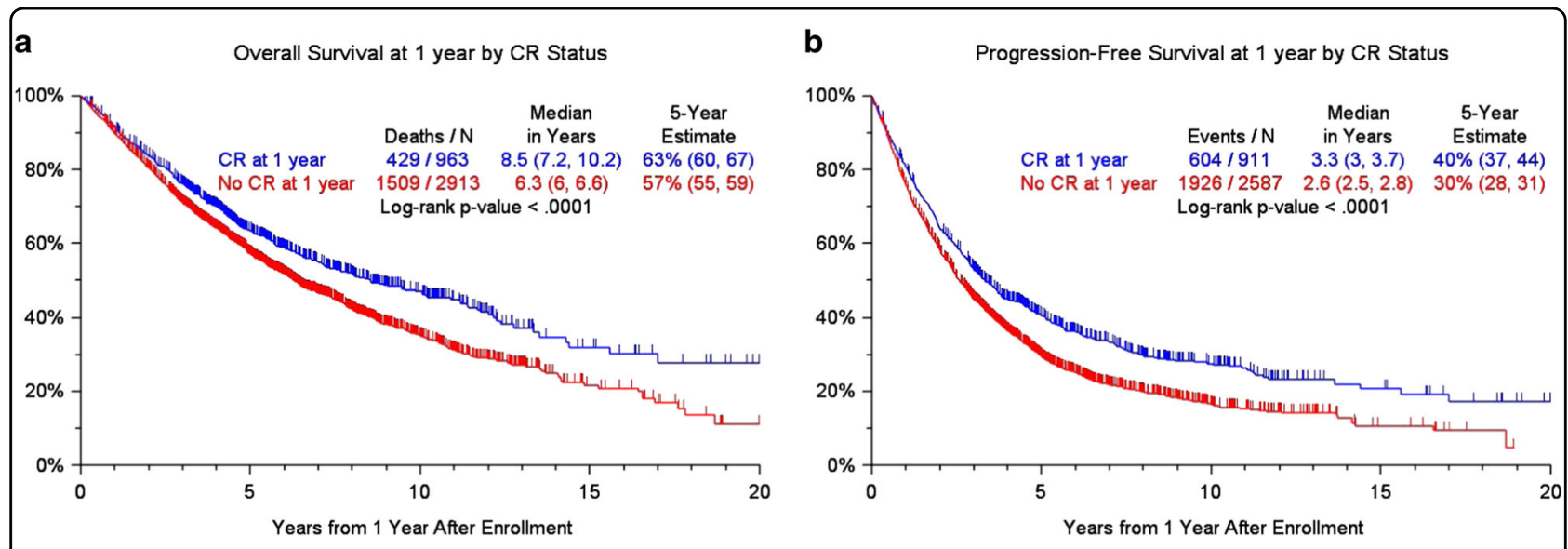

Fig. 1 Overall survival and progression-free survival at 1 year by CR status

Table 1 Patient characteristics by CR Indicator at 1 year

\begin{tabular}{|c|c|c|c|c|c|}
\hline Factor & All patients & $\begin{array}{l}\text { Less than } C R \text { within first } \\
\text { year }\end{array}$ & CR within first year & $P$-value & $\begin{array}{l}\text { Percent of patients } \mathbf{w} / \\
\text { data }\end{array}$ \\
\hline Age at registration $>=65 \mathrm{yr}$ & 258/3867 (7\%) & 194/2906 (7\%) & $64 / 961(7 \%)$ & 0.986 & 258/3867 (7\%) \\
\hline $\lg A$ & 735/3086 (24\%) & 499/2326 (21\%) & $236 / 760(31 \%)$ & $<0.001$ & $735 / 3086(24 \%)$ \\
\hline $\lg G$ & 1868/3086 (61\%) & $1549 / 2326(67 \%)$ & $319 / 760(42 \%)$ & $<0.001$ & 1868/3086 (61\%) \\
\hline Female & $1380 / 3286(42 \%)$ & $1018 / 2442(42 \%)$ & $362 / 844(43 \%)$ & 0.542 & $1380 / 3286(42 \%)$ \\
\hline Albumin $<3.5 \mathrm{~g} / \mathrm{dL}$ & $1041 / 3443(30 \%)$ & $819 / 2590(32 \%)$ & 222/853 (26\%) & 0.002 & $1041 / 3443(30 \%)$ \\
\hline $\mathrm{B} 2 \mathrm{M}>=3.5 \mathrm{mg} / \mathrm{L}$ & $1517 / 3681(41 \%)$ & 1126/2753 (41\%) & $391 / 928(42 \%)$ & 0.510 & 1517/3681 (41\%) \\
\hline$B 2 M>5.5 \mathrm{mg} / \mathrm{L}$ & 708/3681 (19\%) & $517 / 2753(19 \%)$ & 191/928 (21\%) & 0.231 & $708 / 3681(19 \%)$ \\
\hline Creatinine $>=2 \mathrm{mg} / \mathrm{dL}$ & $397 / 3802(10 \%)$ & $282 / 2852(10 \%)$ & $115 / 950(12 \%)$ & 0.056 & $397 / 3802(10 \%)$ \\
\hline $\mathrm{HGB}<10 \mathrm{~g} / \mathrm{dL}$ & $1280 / 3785(34 \%)$ & $973 / 2842(34 \%)$ & $307 / 943(33 \%)$ & 0.343 & $1280 / 3785(34 \%)$ \\
\hline LDH > Upper Limit Normal & $403 / 2244(18 \%)$ & 288/1740 (17\%) & $115 / 504(23 \%)$ & 0.002 & $403 / 2244(18 \%)$ \\
\hline Platelet Count $<150 \times 10^{\wedge} 9 / \mathrm{L}$ & 635/3758 (17\%) & $454 / 2838(16 \%)$ & 181/920 (20\%) & 0.011 & 635/3758 (17\%) \\
\hline $\begin{array}{l}\text { Any clonal abnormality by } \\
\text { cytogenetics }\end{array}$ & $322 / 1256(26 \%)$ & 209/822 (25\%) & 113/434 (26\%) & 0.814 & $322 / 1256(26 \%)$ \\
\hline $\mathrm{BMPC}>=30 \%$ & 1986/3077 (65\%) & $1472 / 2260(65 \%)$ & $514 / 817(63 \%)$ & 0.257 & 1986/3077 (65\%) \\
\hline ISS Stage 1 & 1860/2645 (70\%) & 1351/1949 (69\%) & 509/696 (73\%) & 0.057 & 1860/2645 (70\%) \\
\hline ISS Stage 2 & $1505 / 3302(46 \%)$ & $1121 / 2475(45 \%)$ & $384 / 827$ (46\%) & 0.569 & 1505/3302 (46\%) \\
\hline ISS Stage 3 & $1242 / 3388(37 \%)$ & $954 / 2542(38 \%)$ & 288/846 (34\%) & 0.067 & $1242 / 3388(37 \%)$ \\
\hline
\end{tabular}

$n / N(\%): n-N u m b e r$ with factor, $N$-Number with valid data for factor

$N D$ No valid observations for factor

${ }^{*} P$-value from Fisher's exact test, otherwise chi-squared test. $P$-values represent a comparison between groups, not against the overall population

$p<0.001$ ), BM plasmacytosis $<30 \%$ (OR 2.31, $p<0.001$ ), and ISS stage I or II (OR 3.59, $p<0.001)$ appeared to be positively associated with 10 -year survival. In multivariate analysis, age $\leq 65$ years (OR $1.87, p=0.002$ ), non-IgA Isotype (OR 1.53, $p=0.004$ ), normal albumin $<3.5 \mathrm{~g} / \mathrm{dL}$ $(\mathrm{OR}=1.36, \quad p=0.023)$, low beta 2 microglobulin $\leq$ $3.5 \mathrm{mg} / \mathrm{dl}$ (OR 1.86, $p<0.001$ ), serum creatinine levels $<$ $2 \mathrm{mg} / \mathrm{dL}$ (OR 1.77, $p=0.005$ ), hemoglobin levels $\geq 10 \mathrm{~g} /$
$\mathrm{dL}$ (OR $1.55, p=0.003)$, and platelet count $\geq 150 \mathrm{k} / \mu \mathrm{L}$ (OR $2.26, p<0.001)$ appeared to positively associated with 10 year survival. Interestingly, cytogenetic abnormalities did not feature in multivariate analysis.

We then examined the RS of the whole patient cohort to matched general population. We note that RS was between 0.8 and 0.95 for all countries during the first several years of therapy, with RS for all countries 
Table 2 Logistic regression, 10-year survival vs. 2-year death

\begin{tabular}{|c|c|c|c|c|c|}
\hline \multirow[b]{2}{*}{ Variable } & \multicolumn{5}{|c|}{ Factors differentiating 10 -year survival vs. 2 -year death } \\
\hline & $N$ & Survival less than 2 years & Survival more than 10 years & OR $(95 \% \mathrm{Cl})$ & $P$-value \\
\hline \multicolumn{6}{|l|}{ Univariate } \\
\hline Age at registration $>=65 \mathrm{yr}$ & 2374 & $163 / 214(76 \%)$ & $1271 / 2160(59 \%)$ & $2.24(1.61,3.10)$ & $<0.001$ \\
\hline $\lg A$ & 1878 & $306 / 463(66 \%)$ & $785 / 1415(55 \%)$ & $1.56(1.26,1.95)$ & $<0.001$ \\
\hline $\lg G$ & 1878 & $580 / 1080(54 \%)$ & $511 / 798(64 \%)$ & $0.65(0.54,0.79)$ & $<0.001$ \\
\hline Female & 1918 & $493 / 827(60 \%)$ & 685/1091 (63\%) & $0.87(0.73,1.05)$ & 0.157 \\
\hline Albumin $<3.5 \mathrm{~g} / \mathrm{dL}$ & 2206 & $559 / 808(69 \%)$ & 737/1398 (53\%) & $2.01(1.68,2.42)$ & $<0.001$ \\
\hline $\mathrm{B} 2 \mathrm{M}>=3.5 \mathrm{mg} / \mathrm{L}$ & 2208 & $833 / 1123(74 \%)$ & $488 / 1085(45 \%)$ & $3.51(2.94,4.20)$ & $<0.001$ \\
\hline $\mathrm{B} 2 \mathrm{M}>5.5 \mathrm{mg} / \mathrm{L}$ & 2208 & $501 / 630(80 \%)$ & $820 / 1578(52 \%)$ & $3.59(2.89,4.46)$ & $<0.001$ \\
\hline Creatinine $>=2 \mathrm{mg} / \mathrm{dL}$ & 2320 & $353 / 430(82 \%)$ & 1042/1890 (55\%) & $3.73(2.87,4.85)$ & $<0.001$ \\
\hline $\mathrm{HGB}<10 \mathrm{~g} / \mathrm{dL}$ & 2313 & $678 / 908(75 \%)$ & $699 / 1405(50 \%)$ & $2.98(2.48,3.57)$ & $<0.001$ \\
\hline LDH > Upper Limit Normal & 935 & 196/260 (75\%) & $354 / 675(52 \%)$ & $2.78(2.02,3.82)$ & $<0.001$ \\
\hline Platelet Count $<150 \times 10^{\wedge} 9 / \mathrm{L}$ & 2241 & $364 / 443(82 \%)$ & $969 / 1798(54 \%)$ & $3.94(3.04,5.11)$ & $<0.001$ \\
\hline Any clonal abnormality by cytogenetics & 507 & $92 / 145(63 \%)$ & 127/362 (35\%) & $3.21(2.15,4.80)$ & $<0.001$ \\
\hline $\mathrm{BMPC}>=30 \%$ & 1933 & $800 / 1226(65 \%)$ & $317 / 707$ (45\%) & $2.31(1.91,2.79)$ & $<0.001$ \\
\hline ISS Stage 1 & 2070 & 296/761 (39\%) & $910 / 1309(70 \%)$ & $0.28(0.23,0.34)$ & $<0.001$ \\
\hline ISS Stage 2 & 2105 & $477 / 756(63 \%)$ & $759 / 1349(56 \%)$ & $1.33(1.11,1.60)$ & 0.002 \\
\hline ISS Stage 3 & 2208 & $501 / 630(80 \%)$ & $820 / 1578(52 \%)$ & $3.59(2.89,4.46)$ & $<0.001$ \\
\hline \multicolumn{6}{|l|}{ Multivariate (stratified by country) } \\
\hline Age at registration $>=65 \mathrm{yr}$ & 1230 & 98/144 (68\%) & $525 / 1086(48 \%)$ & $1.87(1.26,2.79)$ & 0.002 \\
\hline $\lg A$ & 1230 & 180/298 (60\%) & 443/932 (48\%) & $1.53(1.15,2.04)$ & 0.004 \\
\hline Albumin $<3.5 \mathrm{~g} / \mathrm{dL}$ & 1230 & $294 / 474(62 \%)$ & $329 / 756(44 \%)$ & $1.36(1.04,1.78)$ & 0.023 \\
\hline $\mathrm{B} 2 \mathrm{M}>=3.5 \mathrm{mg} / \mathrm{L}$ & 1230 & $376 / 569(66 \%)$ & $247 / 661(37 \%)$ & $1.86(1.41,2.45)$ & $<0.001$ \\
\hline Creatinine $>=2 \mathrm{mg} / \mathrm{dL}$ & 1230 & 130/174 (75\%) & 493/1056 (47\%) & $1.77(1.18,2.65)$ & 0.005 \\
\hline $\mathrm{HGB}<10 \mathrm{~g} / \mathrm{dL}$ & 1230 & $288 / 429(67 \%)$ & $335 / 801$ (42\%) & $1.55(1.16,2.06)$ & 0.003 \\
\hline Platelet Count $<150 \times 10 \wedge 9 / \mathrm{L}$ & 1230 & $163 / 218(75 \%)$ & $460 / 1012(45 \%)$ & $2.26(1.59,3.22)$ & $<0.001$ \\
\hline
\end{tabular}

combined close to 0.9 , the ISS III patients had a lower RS compared to ISS I/II patients (Fig. 2a). Cumulative RS plots by ISS staging also shows a decline over time in the ISS III patients' probability of achieving RS similar to matched population (Fig. 2b). The RS curves were also examined by country (Supplemental Figures 3a-h) and showed similar trends to the overall dataset. The statistical cure fraction for the whole group appears to be $14.3 \%$ (Fig. 2c), which signifies the overall proportion of MM patients in this cohort who were able to achieve or exceed expected survival compared to matched general population.

\section{Discussion}

There have been several large population based studies that have highlighted the trends of improvements in MM survival and outcomes ${ }^{11-14}$. Whereas we can attribute this success to the therapeutic success of new drug classes, it also clear that not every MM patient achieves the same depth of response. We also appreciate that despite achieving a good depth of by current IMWG criteria, a proportion of $\mathrm{MM}$ patients remains at a high risk of relapse within the first 2 years of diagnosis ${ }^{15}$. It has also been observed that, despite not achieving $\mathrm{CR}$, patients with documented antecedent smoldering course and 

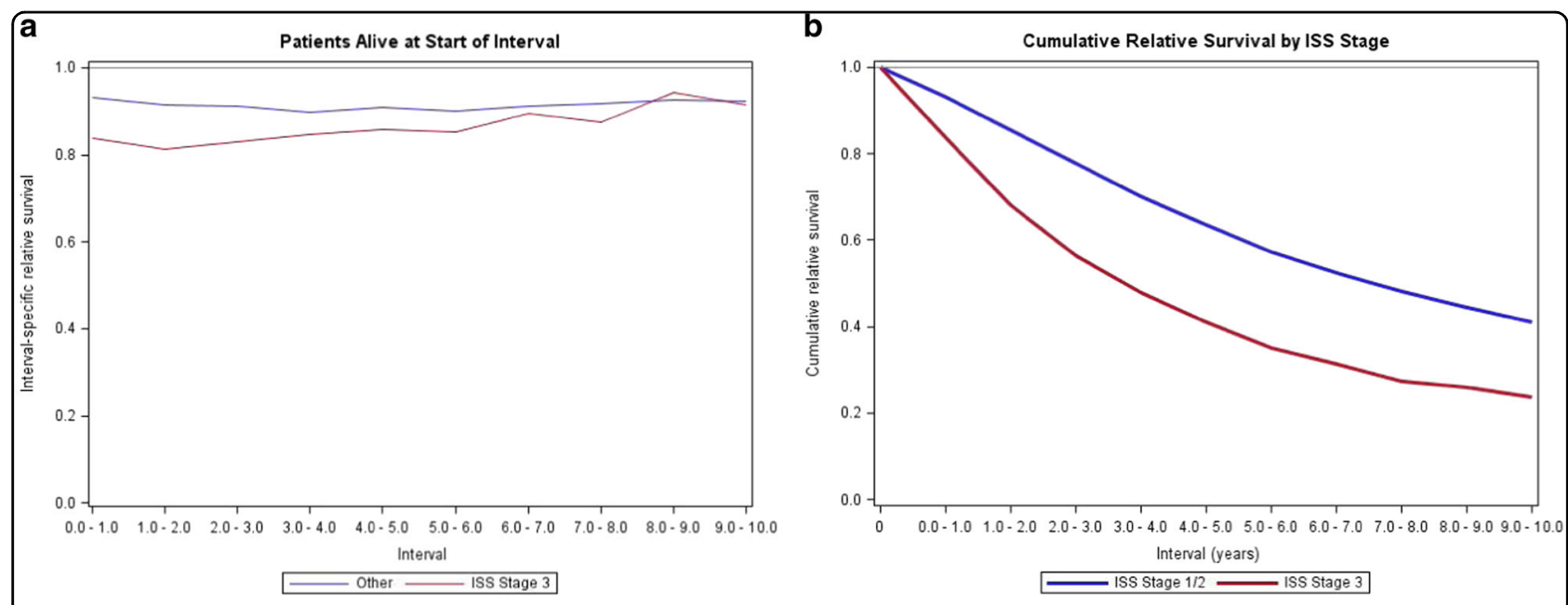

C

All Patients

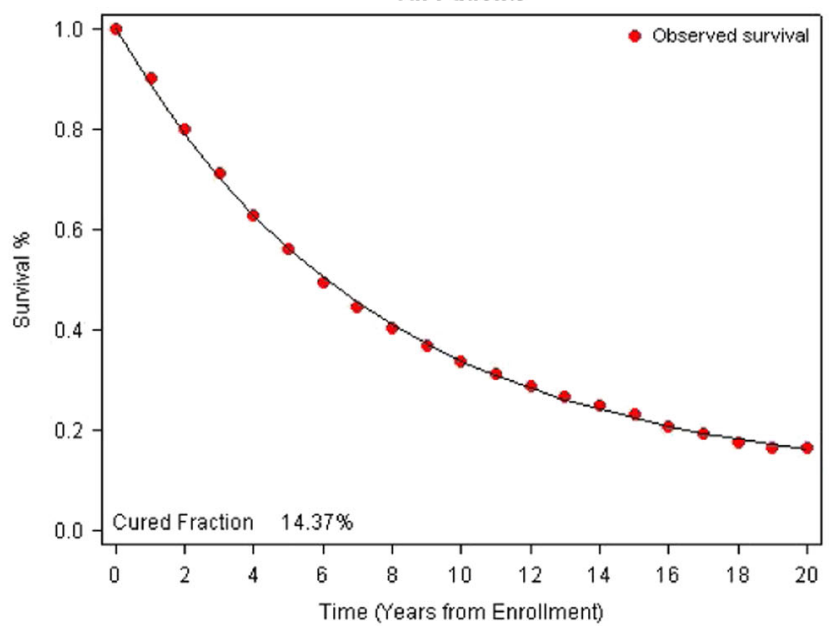

Fig. 2 Relative Survival Analyses (A) Relative survival, ISS Stage III vs. ISS Stage I/II, (B) Cumulative relative survival, ISS Stage III vs. ISS Stage I/II, (C) Cure fraction for the study population

those presenting with a MGUS-like gene expression profiling (GEP) signature can have OS of over 10 years (75\% patients) after HDM-ASCT ${ }^{16}$. This perhaps explains the subgroup of PR patients in the Spanish Myeloma group experience that remain progression free at 12years ${ }^{7}$.

Several next generation sequencing studies in $\mathrm{MM}^{17,18}$ have elegantly demonstrated the heterogeneous nature of MM, even within the same patient during the course of disease, when treated sequentially with novel therapies. These data highlight the genetic chaos that is present from the very onset of disease and further evolves rapidly with each relapse so that eventually total resistance to salvage interventions ensues. We also have to appreciate that there are perhaps more effective drugs and drug classes in the clinician's armamentarium than was available for MM patients being treated in the 1990s or even early 2000s. This may mean that the depth of response after induction therapy may continue to improve over time, potentially further improving the PFS/OS of biologic subset who previously achieved PR yet had good longterm survival.

Cure modeling has been utilized for survival analysis for over three decades. In general, survival analyses include censored observations for subjects who are lost to followup or have not experienced and event at the time the analysis is conducted. Most cure modeling has been done in pediatric cancer population, where the other causes of death can be ignored on account of rarity. In case of adult cancer, there may be subjects who never have disease recurrence and perhaps have been cured of the disease. Cure modeling is a special statistical technique which attempts to capture this subset of patients and several different techniques such as cure fractions and RS rate have been described in literature ${ }^{19}$. To calculate cure fraction, one has to assume that the disease is cured and 
that it is difficult to make this technique applicable to the whole MM population due to competing causes of death -but perhaps the younger, HDM-ASCT eligible MM patients may benefit from such an analysis ${ }^{20}$. In the present analysis, we see a cure fraction of $14.37 \%$ for the study population which is impressive; yet we must bear in mind that this is a select, HDM-ASCT eligible MM patient population on clinical trials. It is important to emphasize that, in order to have a mature long-term follow-up, most patients included in the present study were treated with conventional agents before the era of novel drugs.

To adjust for the lack of evidence of cure for individual patients, a better approach may be looking at "statistical" or "functional" cure via RS modeling. This latter technique compares the expected survival of a given subjects with similar characteristics and co-morbidities to the actual survival as a result of cancer. The expected survival is readily available through life tables available through national mortality statistics. For most cancers, it takes several years before the RS curve reach a plateau. We observe a similar trend for HDM-ASCT eligible MM patients in the current analysis, where there is steady rise in the RS beyond 5 years of diagnosis regardless of ISS staging.

The present study is the largest cohort of HDM-ASCT eligible patients that has been collected to interrogate the clinical predictors of long-term survivors. An important caveat in the current study is that the dataset includes HDM-ASCT eligible MM patients enrolled on clinical trials. Choosing this cohort afforded us completeness of baseline clinical variables, availability of long-term followup and survival data. We identified achieving CR in the first year of diagnosis an important landmark as it was found to be associated with superior PFS and OS. This is an important finding and underscores the importance of depth of response as we explore novel regimens for newly diagnosed MM along with MRD endpoints. Next, the study identified older age at diagnosis and higher burden of disease (by BM plasmacytosis, level of anemia and serum creatinine levels, etc.) adversely affected probability of long-term survival ( $>10$ years) compared with early death $(<2$ years). Although high risk features such as elevated serum LDH levels and having any cytogenetics abnormalities were adverse variables in univariate analysis, they did not feature in multivariate analysis. This perhaps is a function of having inadequate cytogenetics information on all the patients in the dataset and reliance on conventional band karyotyping.

\footnotetext{
Author details

${ }^{1}$ Hematogic Oncology and Blood Disorders, Levine Cancer Institute/Atrium Health, Charlotte, NC, United States. ${ }^{2}$ Cancer Research and Biostatistics, Seattle, Washington, United States. ${ }^{3}$ Seragnoli Institute of Hematology, Bologna University School of Medicine, Bologna, Italy. ${ }^{4}$ Centro de Investigación Médica
}

Aplicada, IDISNA, CIBERONC, Clinica Universidad de Navarra, Pamplona, Spain. ${ }^{5}$ Department of Internal Medicine V, University Hospital Heidelberg, Heidelberg, Germany. ${ }^{6}$ University Hospital Ostrava and the Faculty of Medicine, CRAB, University of Ostrava Adam Rosenthal, Seattle, United States.

${ }^{7}$ Department of Hematology, Malmo University Hospital, Malmo, Sweden. ${ }^{8}$ Hospital Universitario 12 de Octubre, Madrid, Spain. ${ }^{9}$ Service d'Hématologie, CHU de Toulouse-Institut Universitaire du Cancer de Toulouse Oncopole, Toulouse, France. ${ }^{10}$ Hematology, Mount Sinai University, New York, United States. " Gachon University Gil Medical Center, Gachon University School of Medicine, Incheon, Republic of Korea. ${ }^{12}$ Department of Internal Medicine, Division of Hematology, Mayo clinic, Rochester, MN, United States. ${ }^{13}$ Skane University Hospital, Lund, Sweden. ${ }^{14}$ MIRT, UAMS, Myeloma Insitute, Little Rock, United States. ${ }^{15}$ Division of Hematology, Mayo Clinic, Rochester, MN, United States. ${ }^{16}$ Hematology/Oncology, Samuel Oschin Comprehensive Cancer Institute, Cedars-Sinai Medical Center, Los Angeles, United States. ${ }^{17}$ service d'Hematologie, CHU de Nantes, Nantes, France

\section{Conflict of Interest}

SZU reports consulting for Abbvie, Amgen, BMS, Celgene, Janssen, Takeda, Sanofi and SkylineDx; speaker's fees for Amgen, Celgene, Janssen and Takeda; and research funding from Amgen, Array Biopharma, BMS, Celgene, Janssen, Pharmacyclics, Sanofi, Takeda.

\section{Publisher's note}

Springer Nature remains neutral with regard to jurisdictional claims in published maps and institutional affiliations.

Supplementary Information accompanies this paper at (https://doi.org/ 10.1038/s41408-018-0155-7).

Received: 23 February 2018 Revised: 18 June 2018 Accepted: 5 July 2018 Published online: 23 November 2018

\section{References}

1. Palumbo, A. et al. Oral melphalan, prednisone, and thalidomide in elderly patients with multiple myeloma: updated results of a randomized controlled trial. Blood 112, 3107-3114 (2008).

2. Palumbo, A. et al. Melphalan, prednisone, and lenalidomide treatment for newly diagnosed myeloma: a report from the GIMEMA-Italian Multiple Myeloma Network. J. Clin. Oncol. 25, 4459-4465 (2007).

3. San Miguel, J. F. et al. Bortezomib plus melphalan and prednisone for initial treatment of multiple myeloma. N. Engl. J. Med. 359, 906-917 (2008).

4. Mateos, M. V. et al. Bortezomib, melphalan, and prednisone versus bortezomib, thalidomide, and prednisone as induction therapy followed by maintenance treatment with bortezomib and thalidomide versus bortezomib and prednisone in elderly patients with untreated multiple myeloma: a randomized trial. Lancet Oncol. 11, 934-941 (2010).

5. Usmani, S. Z. et al. Improvement in long-term outcomes with successive Total Therapy trials for multiple myeloma: are patients now being cured? Leukemia 27. 226-232 (2013).

6. Martinez-Lopez, J. et al. Prognostic value of deep sequencing method for minimal residual disease detection in multiple myeloma. Blood 123, 3073-3079 (2014)

7. Martinez-Lopez, J. et al. Long-term prognostic significance of response in multiple myeloma after stem cell transplantation. Blood 118, 529-534 (2011).

8. Kaplan, E. L. \& Meier, P. Nonparametric estimation using incomplete observations. J. Am. Stat. Assoc. 53, 457-481 (1958).

9. Dickman, P. W. et al. Regression models for relative survival. Stat. Med. 23, 51-64 (2004)

10. Berkson, J. \& Gage, R. P. Survival curve for cancer patients following treatment. J. Am. Stat. Assoc. 42, 501-515 (1952).

11. Kristinsson, S. Y. et al. Patterns of survival in multiple myeloma: a populationbased study of patients diagnosed in Sweden from 1973 to 2003. J. Clin. Oncol. 25, 1993-1999 (2007).

12. Turesson, I., Velez, R., Kristinsson, S. Y. \& Landgren, O. Patterns of improved survival in patients with multiple myeloma in the twenty-first century: a population-based study. J. Clin. Oncol. 28, 830-834 (2010). 10. 
13. Brenner, H., Gondos, A. \& Pulte, D. Expected long-term survival of patients diagnosed with multiple myeloma in 2006-2010. Haematologica 94, 270-275 (2009).

14. Pulte, D. et al. Trends in survival of multiple myeloma patients in Germany and the United States in the first decade of the 21st century. Br. J. Haemato/ 171, 189-196 (2015).

15. Usmani, S. Z. et al. Defining and treating high-risk multiple myeloma. Leukemia 29, 2119-2125 (2015).

16. Zhan, F. et al. Gene-expression signature of benign monoclonal gammopathy evident in multiple myeloma is linked to good prognosis. Blood 109, 1692-1700 (2007)
17. Chapman, M. A. et al. Initial genome sequencing and analysis of multiple myeloma. Nature 471, 467-472 (2011).

18. Egan, J. B. et al. Whole-genome sequencing of multiple myeloma from diagnosis to plasma cell leukemia reveals genomic initiating events, evolution, and clonal tides. Blood 120, 1060-1066 (2012).

19. Lambert, P. C. et al. Estimating and modeling cure fractions in populationbased cancer survival analysis. Biostatistics 8, 576-594 (2007).

20. Barlogie, B. et al. Curing myeloma at last: defining criteria and providing the evidence. Blood 124, 3043-3051 (2014). 